\title{
Construction status and prospects of the Hyper-Kamiokande project
}

\section{Yoshitaka Itow for Hyper-Kamiokande Collaboration ${ }^{a, *}$}

${ }^{a}$ Institute for Space-Earth Environmental Research / Kobayashi-Maskawa Institute for the Origin of Particles and the Universe, Nagoya University, Nagoya, Aichi, Japan

E-mail: itow@isee.nagoya-u.ac.jp

The Hyper-Kamiokande project is a 258-kton Water Cherenkov together with a 1.3-MW highintensity neutrino beam from the Japan Proton Accelerator Research Complex (J-PARC). The inner detector with 186-kton fiducial volume is viewed by 20 -inch photomultiplier tubes (PMTs) and multi-PMT modules, and thereby provides state-of-the-art of Cherenkov ring reconstruction with thresholds in the range of few MeVs. The project is expected to lead to precision neutrino oscillation studies, especially neutrino $\mathrm{CP}$ violation, nucleon decay searches, and low energy neutrino astronomy. In 2020, the project was officially approved and construction of the far detector was started at Kamioka. In 2021, the excavation of the access tunnel and initial mass production of the newly developed 20-inch PMTs was also started. In this paper, we present a basic overview of the project and the latest updates on the construction status of the project, which is expected to commence operation in 2027.

\footnotetext{
*** 37th International Cosmic Ray Conference (ICRC2021), ***

*** 12-23 July $2021 * * *$

*** Berlin, Germany - Online ***
}

\footnotetext{
*Presenter
} 


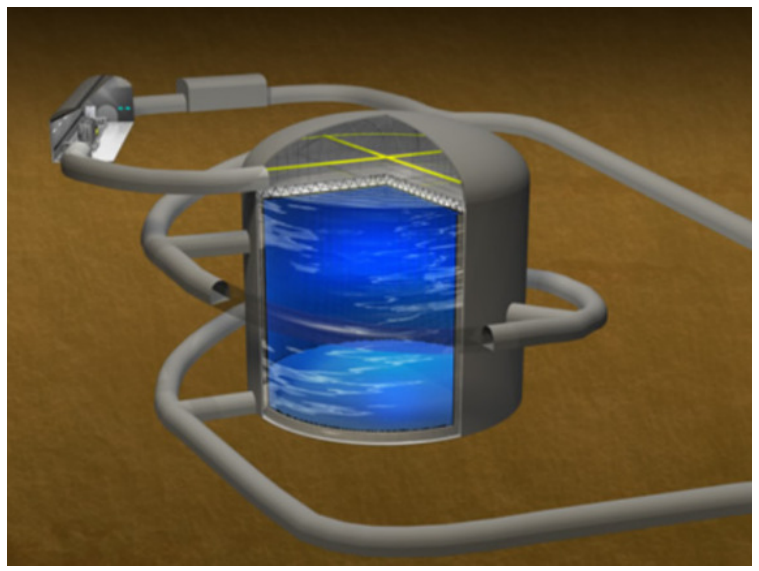

Figure 1: Hyper-Kamiokande detector: a cylindrical tank with a diameter of $68 \mathrm{~m}$ and a height of $71 \mathrm{~m}$ is filled with $258 \mathrm{kt}$ of pure water.

\section{Introduction}

Since the discovery of the evidence of neutrino oscillations in atmospheric neutrinos, there has been significant progress in neutrino physics over the past few decades. The first observation of astrophysical neutrinos from SN1987a and the real-time solar neutrinos have led to a new field of neutrino astronomy. To perform a higher-precision study, a highly massive neutrino detector is desirable. This can substantially improve the current sensitivity limit for nucleon decay searches.

The Hyper-Kamiokande project involves a plan to build the next-generation multi-purpose water Cherenkov detector at Kamioka together with a high-intensity off-axis neutrino beam from the Japan Proton Accelerator Research Complex (J-PARC) at Tokai, Japan, a newly planned near detector, Intermediate Water Cherenkov Detector (IWCD), and existing T2K near detector suits, which will provide initial neutrino flux constraints and a fundamental understanding of neutrino interactions.

The Hyper-Kamiokande far detector is a 258 kton water Cherenkov detector. The detector consists of a cylindrical tank with a diameter of $68 \mathrm{~m}$ and a height of $71 \mathrm{~m}$, as shown in Fig. 1 . The fiducial volume is $188 \mathrm{kton}$, which is eight times larger than that of Super-K, and it is designed to be viewed by high-QE photo-detectors with $40 \%$ photo-coverage. The far detector site is located at Mt. Nijugo-yama in the Tochibora-mine at Kamioka at a distance of $295 \mathrm{~km}$ from J-Parc. At this location approximately $8 \mathrm{~km}$ south of the Super-Kamiokande site, the neutrino beam from J-PARC will be exposed to the same off-axis angle of 2.5 degree, which is identical to as the configuration in the T2K experiment. The rock overburden of the site is approximately $600 \mathrm{~m}$, which is slightly shallower than that for the Super-Kamiokande (approximately $1000 \mathrm{~m}$ ). However, the atmospheric muon rate is still at an affordable level for data collection and analysis.

The goals of Hyper-K physics are precision neutrino oscillation with accelerator neutrinos and neutrinos from natural sources such as atmospheric, sun, or supernova, as shown in Fig. 2. The discovery of neutrino CP violation and precision measurement of neutrino mixing parameters constitute the most urgent subjects. This size of a gigantic water Cherenkov detector will lead to low-energy neutrino astrophysics with high sensitivity and high precision, such as the detection of 

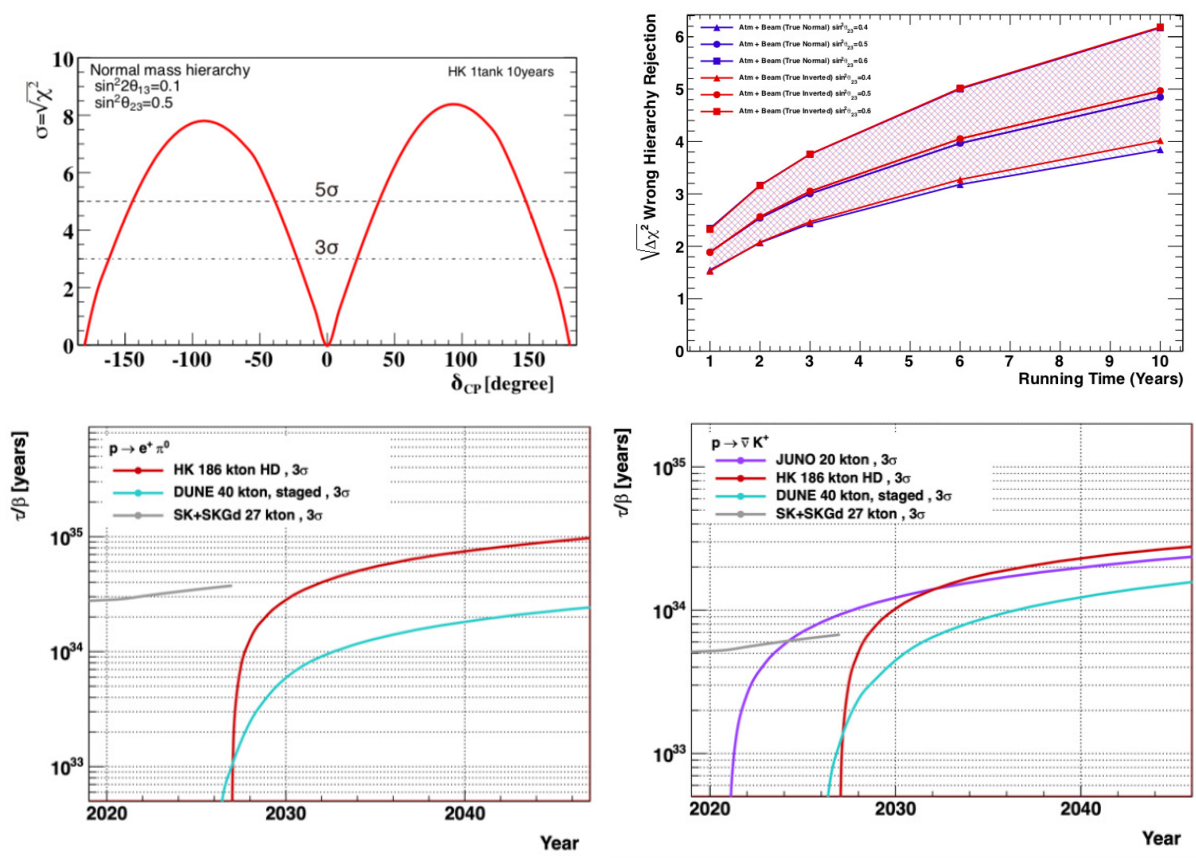

Figure 2: The Hyper-K physics sensitivities. (Upper left) the expected CP violation sensitivity for 10 years operation as a function of $\mathrm{CP} \delta$ with $\sin ^{2} \theta_{13}=0.1$ and $\sin ^{2} \theta_{23}=0.5$. Normal hierarchy is assumed. (Upper right) the wrong hierarchy rejection sensitivity for atmospheric neutrino observation as a function of running time. (Bottom left) the $p \rightarrow e^{+}+\pi^{0}$ life time with 3- $\sigma$ discovery as a function of running time. (bottom right) the $p \rightarrow \bar{v}+K^{+}$life time with 3- $\sigma$ discovery as a function of running time.

solar HEP neutrinos or supernova neutrinos from distant galaxies, such as M31. The first hint of the proton decay can potentially be delivered by such a gigantic water Cherenkov detector with a fiducial mass that is larger than that of current Super-Kamiokande by about one order of magnitude. The 3- $\sigma$ discovery can be realized for $p \rightarrow e^{+} \pi^{0}$ and $p \rightarrow \bar{v} K^{+}$with $10^{35}$ years and $3 \times 10^{34}$ years, respectively. There are rich scientific cases from low energy to high energy. The basic description of the detectors and the projected physics sensitivities can be found elsewhere [1-3].

In 2020, the project was officially approved by the host country. The collaboration transitioned from a proto-collaboration to a real collaboration in September 2020. Accordingly, the collaboration structure is reorganized for the construction work in the coming years. The number of collaborators has been increasing rapidly since the project approval, and it now involves a total of 450 people from 93 institutes in 19 countries participate as of May 2021.

Figure 3 shows the project timeline and milestones. In 2020, the intensive geological surveys and site preparation work were conducted. In 2021, access tunnel excavation was commenced, and it was followed by the excavation of the main cavern from 2023 to 2024. In 2021, mass production of the new 20-inch PMT commenced and was followed by the delivery of electronics and other equipment. The construction of the tank structure is scheduled in 2024 and 2025, and then PMT installation will occur in 2026. Simultaneously, the power upgrade of the neutrino beam began during the shutdown period in 2021 and 2022 at J-Parc. After integrating all the efforts, the experiment will be online in 2027. 


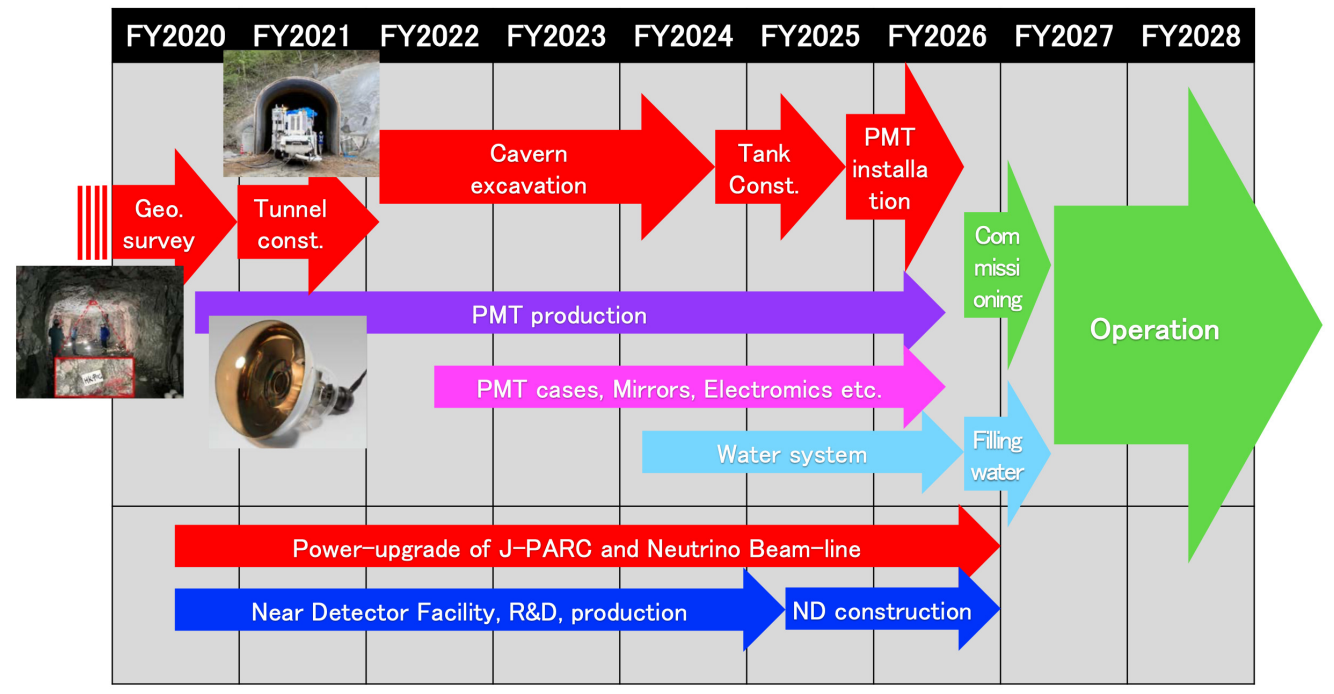

Figure 3: Time line of construction.

In this article, we present basic overview of the project and report the latest updates on the construction status as of the summer of 2021.

\section{Current status of construction works}

In 2020, the construction of the entrance yard and intensive geological surveys were performed. The results indicated that the rock quality at the Hyper-K main cavity location was excellent. The basic design of an underground facility was determined. As a major milestones, the excavation of the access tunnel commenced. The entrance to the Hyper-K underground facility is currently under construction.

Figure 4 (left) shows the potential photo-detector arrangement of the Inner Detector (ID) and the Outer Detector (OD). The ID volume is approximately based on a dimater of $64 \mathrm{~m}$ and height of $66 \mathrm{~m}$, and it is designed to be viewed by photo-detectors with a $40 \%$ photo-coverage.

With respect to ID, a new 20-inch PMT (Hamamatsu R12860) is developed with a high QE photo-cathode and with a large Box \& Line (B\&L) type dynode as shown in Fig. 5 left [4]. Thus, to date, installation of 20,000 new B\&L 20-inch PMTs is planned. This in turn will provide a $20 \%$ photo-coverage. Hence, when compared to the Super-K 20-inch PMT, the performance will significantly improve, and it will lead to twice the photo-detection efficiency with a similar dark rate $(\sim 4 \mathrm{kHz})$, better charge and timing resolution, and almost twice the water-pressure tolerance (1.25 MPa). During the last refurbish work in the summer of 2018, 136 prototype PMTs were installed and operated in the Super-K tank for long-term stability checks. To date, no significant problems have been identified. In 2021, the initial mass production of PMTs started, and the first 1,000 PMTs will be delivered to Kamioka. A detailed inspection is in progress. To prevent chain implosion, 20-inch PMTs will be enclosed in protective covers. Currently three different prototypes are designed, and the final test and design choice will occur shortly. 
The addition of multi-PMT (mPMT) modules is planned to provide additional photo-coverage and to provide new information on Cherenkov photons. The mPMT module consists of 193 -inch PMTs assembled in a pressure vessel with in-case front-end electronics and a high-voltage supply, as shown in Fig. 5 (middle and right) [5]. Each hit at the mPMT will be digitized via the incase front-end electronics and sent to the readout computers. Given the good transit-time-spread, low dark rate of 3-inch PMTs, and new features involving high granularity of photo-coverage and directional information, mPMT can provide unique information for Cherenkov ring reconstruction. For example, it can improve ring reconstruction at the fiducial edge near the wall, and can serve a reference PMT for detector calibration.

The ID is surrounded by OD to veto cosmic ray muons and to shield $\gamma$-rays or neutrons from environmental radioactivity or muon spallation in the rocks. The thickness of barrel OD is $1 \mathrm{~m}$ and that of top/bottom part is $2 \mathrm{~m}$. With the reflection sheets covering the entire surface of the OD region, it will be viewed by 3-inch PMTs with wave length length shifter (WLS) boards to enhance Cherenkov photon yields [6].

To mitigate the disadvantages of large number of excessively long cables from photo-detectors $(\sim 100 \mathrm{~m})$, the front-end electronics and high-voltage supply units will be placed under the water contained in pressure vessels. It is not possible to frequently replace the underwater equipment once a large amount of water is filled. Therefore the overall failure rate must be low enough, i.e., $1 \%$ per year. This constitutes a major challenge in Hyper-K R\&D. Currently, four different digitizer options are proposed, and R\&D work is ongoing [7]. Additionally, a pressure vessel, water-tight feedthrough connectors, and heat dissipation design for underwater front-end electronics are currently being intensively developed.

ID photo-detectors, 20-inch PMTs, and mPMT modules will be placed inward at the $70 \mathrm{~cm}$ $\times 70 \mathrm{~cm}$ grid, while some of the grids will be occupied by underwater electronics vessels. With this hybrid photo-detector configuration, Cherenkov rings can be detected by 20-inch PMTs with additional useful information from mPMT modules as shown in Fig. 4 (right). The ID and OD are optically separated by the opaque sheets. Furthermore, OD photo-detectors with WLS boards will be placed outward and their front-end electronics will also be placed under the water in a manner similar to that of 20-inch PMTs.

All the raw hits of the photo-detectors are digitized at the underwater front-end electronics, and then digitized data will then be sent to the readout computers from the underwater box via an optical link. All the read out hits are buffered by the Read Out Buffer Unit running in the online computers. Subsequently, they will be processed by the Trigger Processor Unit to issue the triggers based on the purposes and then they will be recorded.

At the J-Parc site, upgrade works of neutrino beam intensity have commenced. Beam upgrade can be mainly realized by increasing the repetition rate of the accelerator, from $2.48 \mathrm{~s}$ per cycle to $1.16 \mathrm{~s}$ per cycle. Furthermore, upgrade works, including that of magnet power supply, is scheduled in the 2-years shutdown period in 2021-2022. This is followed by various works to manage highradiation environments. In conjunction with RF system upgrade, total neutrino beam power will be as high as $1.3 \mathrm{MW}$ in 2028.

There are near detector suites for monitoring a neutrino beam and studying neutrino interactions at the J-Parc site [8]. The on-axis neutrino direction monitor INGRID and the ND280 magnetized tracker are currently operated as T2K near detectors as shown in Fig 6. The T2K Collaboration is 


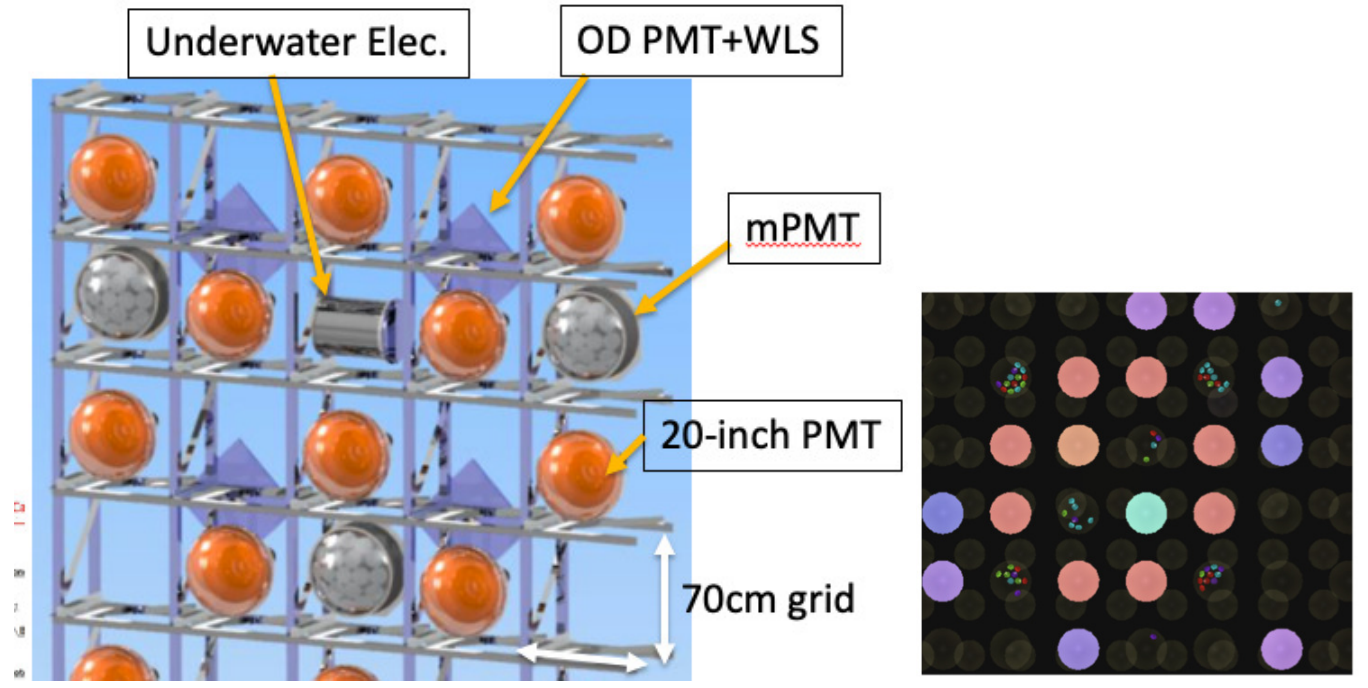

Figure 4: (Left) Hybrid photo-detector arrangement in the far detector. (Right) Cherenkov rings detected by the hybrid configuration.
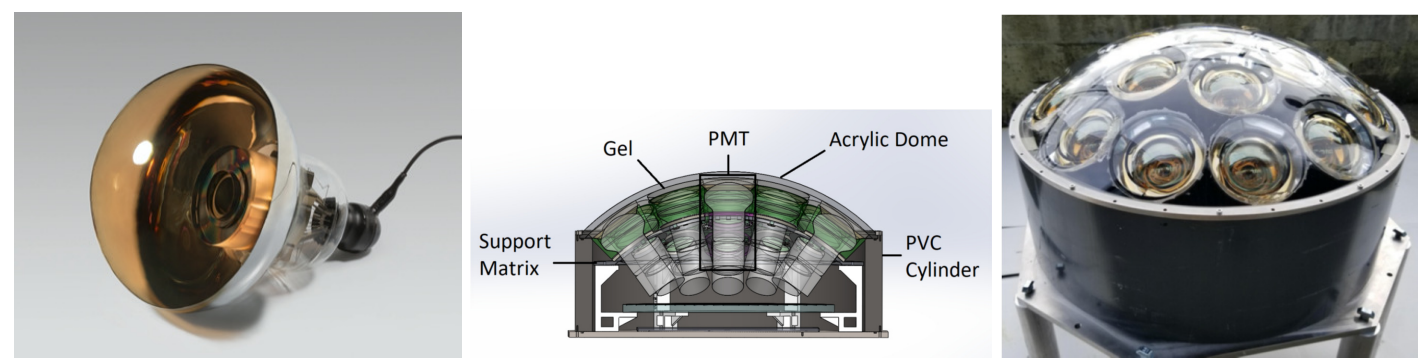

Figure 5: Photo-detectors for the far detector. (Left) a new Box\&Line 20-inch PMT (Hamamatsu R12860), (Middle) schematic view of a multi-PMT module, and (Right) image of a multi-PMT module.

now upgrading ND280 to introduce the Super Fine Grain Detector and new horizontal TPC during the shut down in order to add 3-dimensional tracking capability and to enhance the large angle acceptance. Additionally, the Intermediate Water Cherenkov Detector (IWCD) is planned to be newly built. It will provide neutrino-water interaction, especially electron-neutrino interactions, by using the same technique as that utilized in the Hyper-K far water Cherenkov detector. The water tank of the IWCD can move vertically and scan the off-axis angle of the neutrino beam such that neutrino interactions can be investigated with the different beam energy spectra. It is essential to precisely understand systematic uncertainty in neutrino interactions by considering energy dependence.

\section{Summary}

The Hyper-Kamiokande project is a next-generation multi-purpose neutrino experiment. It will use a 258-kt water Cherenkov detector together with an upgraded 1.3-MW off-axis muon neutrino 

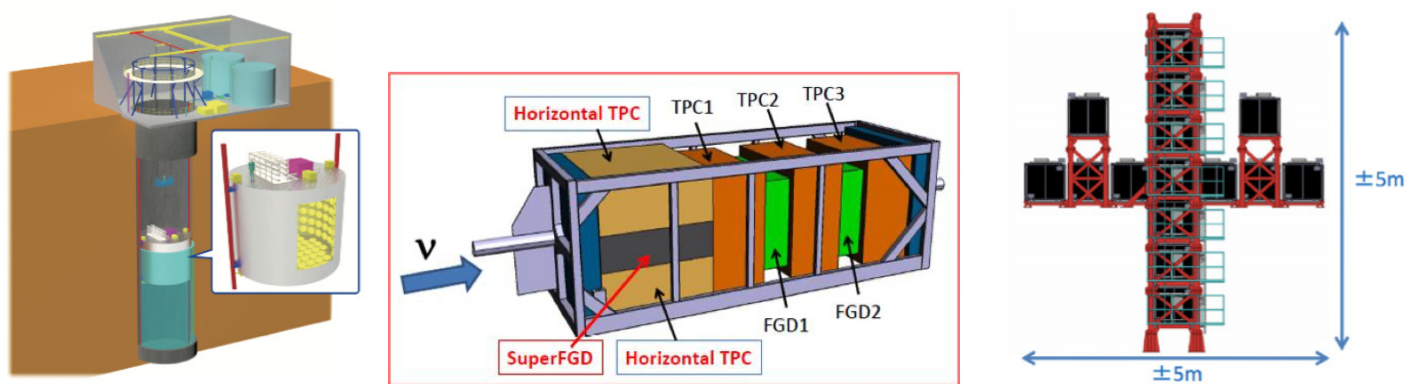

Figure 6: Near detector suites that are expected to be used for the Hyper-K project [8]. (Left) Intermediate Water Cherenkov Detector (IWCD). (Middle) the upgraded T2K ND280 magnetized tracker. (Right) T2K INGRID neutrino beam monitor. ND280 and INGRID are built and operated by the T2K Collaboration.

beam from J-Parc. The project was officially approved in 2020 and subsequently construction was commenced. In 2021, two important milestones have been achieved: excavation of access tunnel and mass production of 20-inch PMTs. The main cavity excavation and tank construction will soon follow. PMT installation is expected to occur in 2025, and the experiment is expected to be online in 2027 along with the upgraded neutrino beam. As soon as the experiment is operational and starts to provide new data, many breakthrough results in the various areas of particle physics and astroparticle physics will be expected.

\section{References}

[1] K. Abe et al. [Hyper-Kamiokande Collaboration], arXiv:1805.04163 [physics.ins-det].

[2] M. Scott et al. [Hyper-Kamiokande Collaboration], PoS ICHEP2020 (2021) 174.

[3] T. Yano et al. [Hyper-Kamiokande Collaboration], PoS ICHEP2020 (2021) 191.

[4] T. Tashiro et al. [Hyper-Kamiokande Collaboration], PoS ICHEP2020 (2021) 876.

[5] G. De Rosa et al. [Hyper-Kamiokande Collaboration], PoS ICHEP2020 (2021) 831.

[6] S. Zsoldos et al. [Hyper-Kamiokande Collaboration], PoS ICHEP2020 (2021) 886.

[7] M. Ziembicki et al. [Hyper-Kamiokande Collaboration], Nucl. Instrum. Meth. A 952 (2020) 161829.

[8] Y. Kudenko et al. [Hyper-Kamiokande Collaboration], JINST 15 (2020) 07, C07029. 


\section{Full Authors List: Collaboration}

K. Abe ${ }^{1,2,3}$, P. Adrich ${ }^{4}$, H. Aihara ${ }^{5,2,3}$, R. Akutsu ${ }^{6}$, I. Alekseev ${ }^{7}$, A. Ali ${ }^{8}$, Y. I. Alj Hakim ${ }^{9}$, F. Ameli ${ }^{10}$, L.H.V. Anthony9 ${ }^{9}$, A. Araya ${ }^{11,3}$, Y. Asaoka ${ }^{1,3}$, V. Aushev ${ }^{12}$, F. Ballester ${ }^{13}$, I. Bandac ${ }^{14}$, M. Barbi ${ }^{15}$, G. Barr ${ }^{16}$, M. Batkiewicz-Kwasniak ${ }^{17}$, M. Bellato ${ }^{18}$, V. Berardi ${ }^{19}$, L. Bernard ${ }^{20}$, E. Bernardini ${ }^{18}$, L. Berns ${ }^{21}$, S. Bhadra ${ }^{22}$, J. Bian ${ }^{23}$, A. Blanchet ${ }^{24}$, A. Blondel ${ }^{24}$, A. Boiano ${ }^{25}$, S. Bolognesi ${ }^{26}$, L. Bonavera ${ }^{27}$, N. Booth ${ }^{28}$, S. Bordoni ${ }^{29}$, S. Borjabad ${ }^{14}$, T. Boschi ${ }^{30}$, D. Bose ${ }^{31}$, S .B. Boyd ${ }^{32}$, C. Bozza ${ }^{33}$, A. Bravar ${ }^{29}$, C. Bronner ${ }^{1,3}$, L. Brown ${ }^{28}$, A. Bubak ${ }^{34}$, A. Buchowicz ${ }^{35}$, M. Buizza Avanzini ${ }^{20}$, F. S. Cafagna ${ }^{19}$, N. F. Calabria ${ }^{36}$, J. M. Calvo-Mozota ${ }^{14}$, S. Cao ${ }^{37,38}$, A. Carroll ${ }^{39}$, M. G. Catanesi ${ }^{19}$, S. Cebriànn ${ }^{40}$, S. Chakraborty ${ }^{41}$, J. H. Choi ${ }^{42}$, S. Choubey ${ }^{43}$, M. Cicerchia ${ }^{44}$, J. Coleman ${ }^{39}$, G. Collazuol ${ }^{18}$, S. Cuen-Rochin" ${ }^{45,6}$, M. Danilov ${ }^{7}$, G. Díaz López ${ }^{46}$, E. De la Fuente ${ }^{47,48,49}$, P. de Perio ${ }^{6}$, G. De Rosa ${ }^{36}$, T. Dealtry ${ }^{50}$, C. J. Densham ${ }^{51}$, A. Dergacheva ${ }^{52}$, N. Deshmukh ${ }^{53}$, M. M. Devi ${ }^{54}$, F. Di Lodovico ${ }^{30}$, P. Di Meo ${ }^{25}$, I. Di Palma ${ }^{10}$, T. A. Doyle ${ }^{50}$, E. Drakopoulou ${ }^{55}$, O. Drapier ${ }^{20}$, J. Dumarchez ${ }^{24}$, L. Eklund ${ }^{56}$, S. El Hedri ${ }^{20}$, J. Ellis ${ }^{30}$, S. Emery ${ }^{26}$, A. Esmaili ${ }^{57}$, R. Esteve ${ }^{13}$, S. Fedotos ${ }^{52}$, J. Feng ${ }^{8}$, P. Fernandez ${ }^{39}$, E. Fernández-Martinez ${ }^{58}$, P. Ferrario ${ }^{59}$, B. Ferrazzi ${ }^{15}$, A. Finch ${ }^{50}$, C. Finley ${ }^{60}$, G. Fiorillo ${ }^{36}$, M. Fitton ${ }^{51}$, M. Friend ${ }^{37,38}$, Y. Fujii ${ }^{37,38}$, C. Fujisawa ${ }^{61}$, Y. Fukuda ${ }^{62}$, G. Galinski ${ }^{35}$, J. Gao $^{30}$, C. Garde ${ }^{53}$, A. Garfagnini ${ }^{18}$, S. Garode ${ }^{53}$, L. Gialanella ${ }^{63}$, C. Giganti ${ }^{24}$, J. J. Gomez-Cadenas ${ }^{59}$, M. Gonin ${ }^{20}$, J. González-Nuevo ${ }^{27}$, A. Gorin ${ }^{52}$, R. Gornea ${ }^{64}$, V. Gousy-Leblanc ${ }^{28}$, F. Gramegna ${ }^{44}$, M. Grassi ${ }^{18}$, G. Grella ${ }^{33}$, M. Guigue ${ }^{24}$, D. R. Hadley ${ }^{32}$, M. Harada ${ }^{65}$, M. Hartz ${ }^{2,6,3}$, S. Hassani ${ }^{26}$, N. C. Hastings ${ }^{37,38}$,

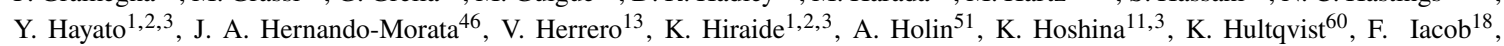
A. K. Ichikawa ${ }^{66}$, W. Idrissi Ibnsalih ${ }^{63}$, K. Ieki ${ }^{1}$, M. Ikeda ${ }^{1,2,3}$, A. Ioannisian ${ }^{67}$, T. Ishida ${ }^{37,38}$, K. Ishidoshiro ${ }^{68}$, H. Ishino ${ }^{65}$, M. Ishitsuka ${ }^{69}$, H. T. Israel ${ }^{70}$, H. Ito ${ }^{1}$, Y. Itow ${ }^{71,72}$, N. Izumi ${ }^{69}$, S. Izumiyama ${ }^{21}$, M. Jakkapu ${ }^{37,73}$, B. Jamieson ${ }^{74}$, J. S. Jang ${ }^{75}$, H. S. Jo ${ }^{76}$, P. Jonsson ${ }^{9}$, K. K. Joo ${ }^{77}$, T. Kajita ${ }^{49,2,3}$, H. Kakuno ${ }^{78}$, J. Kameda ${ }^{1,2,3}$, R. Kaneshima ${ }^{1}$, Y. Kano ${ }^{11,3}$, D. Karlen ${ }^{28,6}$, Y. Kataoka ${ }^{1,3}$, A. Kato ${ }^{11,3}$, T. Katori ${ }^{30}$, N. Kazarian ${ }^{67}$, M. Khabibullin ${ }^{52}$, A. Khotjantsev ${ }^{52}$, T. Kikawa ${ }^{8}$, M. Kekic ${ }^{46}$, J. Y. Kim ${ }^{77}$, S. B. Kim ${ }^{79}$, S. King ${ }^{30}$, T. Kinoshita ${ }^{69}$, J. Kisiel ${ }^{17,34}$, A. Klekotko ${ }^{35}$, T. Kobayashi ${ }^{37,38}$, L. Koerich ${ }^{15}$, N. Kolev ${ }^{15}$, A. Konaka ${ }^{6}$, L. L. Kormos ${ }^{50}$, Y. Koshio ${ }^{65,2}$, Y. Kotsar ${ }^{80}$, K. A. Kouzakov ${ }^{81}$, K.L. Kowalik ${ }^{4}$, L. Kravchuk ${ }^{52}$, A. P. Kryukov ${ }^{81}$, Y. Kudenko ${ }^{52}$,

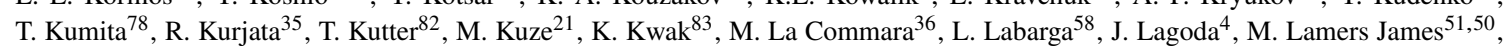

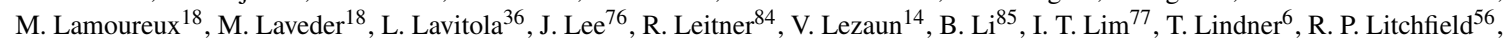
K. R. Long ${ }^{9}$, A. Longhin ${ }^{18}$, P. Loverre ${ }^{10}$, X. Lu ${ }^{16}$, L. Ludovici ${ }^{10}$, Y. Maekawa ${ }^{61}$, L. Magaletti ${ }^{19}$, K. Magar ${ }^{53}$, Y. Makida ${ }^{37,38}$, M. Malek ${ }^{70}$, M. Malinský ${ }^{84}$, T. Marchi ${ }^{44}$, C. Mariani ${ }^{86}$, A. Marinelli ${ }^{25}$, K. Martens ${ }^{2,3}$, Ll. Marti ${ }^{1,3}$, J. F. Martin ${ }^{87}$, D. Martin ${ }^{9}$, J. Marzec ${ }^{35}$, T. Matsubara ${ }^{37,38}$, R. Matsumoto ${ }^{69}$, N. McCauley ${ }^{39}$, A. Medhi ${ }^{54}$, P. Mehta ${ }^{39}$, L. Mellet ${ }^{24}$, H. Menjo ${ }^{88}$, M. Mezzetto ${ }^{18}$, J. Migenda ${ }^{30}$, P. Migliozzi ${ }^{25}$, S. Miki ${ }^{1}$ E. W. Miller ${ }^{30}$, A. Minamino ${ }^{89}$, S. $M_{i n e}^{23}$, O. Mineev ${ }^{52}$, L. Munteanu ${ }^{26}$, A. Mitra ${ }^{32}$, M. Miura ${ }^{1,2,3}$, R. Moharana ${ }^{90}$, C. M. Mollo ${ }^{25}$, T. Mondal ${ }^{*}, 31$, M. Mongelli ${ }^{19}$, F. Monrabal ${ }^{59}$, D. H. Moon ${ }^{77}$, C. S. Moon ${ }^{76}$, F. J. Mora ${ }^{13}$, M. Mori ${ }^{8}$, S. Moriyama ${ }^{1,2,3}$, Th. A. Mueller ${ }^{20}$, T. Nakadaira ${ }^{37,38}$, K. Nakagiri ${ }^{5,3}$, M. Nakahata ${ }^{1,2,3}$, S. Nakai ${ }^{11,3}$, Y. Nakajima ${ }^{5,2,3}$, K. Nakamura ${ }^{37,2}$, KI. Nakamura ${ }^{66}$, H. Nakamura ${ }^{69}$, S. Nakanishi ${ }^{69}$, Y. Nakano ${ }^{80}$, T. Nakaya ${ }^{8,2}$, S. Nakayama ${ }^{1,2,3}$, K. Nakayoshi $^{37,38}$, L. Nascimento Machado ${ }^{36}$, C. E. R. Naseby ${ }^{9}$, B. Navarro-Garcia ${ }^{47}$, M. Needham ${ }^{55}$, K. Niewczas ${ }^{91}$, K. Ninomiya ${ }^{88}$, S. Nishimori ${ }^{37}$, Y. Nishimura ${ }^{61}$, Y. Noguchi ${ }^{8}$, F. Nova ${ }^{51}$, J. C. Nugent ${ }^{56}$, H. Nunokawa ${ }^{57}$, W. Obrebski ${ }^{35}$, J. P. Ochoa-Ricoux ${ }^{23}$, E. O'Connor ${ }^{92}$, N. Ogawa ${ }^{5}$, T. Ogitsu ${ }^{37,38}$, K. Okamoto ${ }^{1}$, H. M. O'Keeffe ${ }^{50}$, K. Okumura $^{49,2,3}$, Y. Onishchuk ${ }^{12}$, F. Orozco-Luna ${ }^{48}$, A. Oshlianskyi ${ }^{12}$, N. Ospina ${ }^{18}$, M. Ostrowski ${ }^{93}$, E. O’Sullivan ${ }^{94}$, Y. Oyama ${ }^{37,38}$, H. Ozaki ${ }^{80}$, M.Y. Pac ${ }^{42}$, P. Paganini ${ }^{20}$, V. Palladino ${ }^{36}$, M. Pari ${ }^{18}$, J. Pasternak ${ }^{9}$, C. Pastore ${ }^{19}$, G. Pastuszak ${ }^{35}$, D. A. Patel ${ }^{15}$, M. Pavin ${ }^{6}$, D. Payne ${ }^{39}$, C. Peña-Garay ${ }^{14}$, C. Pidcott ${ }^{70}$, S. Playfer ${ }^{55}$, B. W. Pointon ${ }^{95,6}$, A. Popov ${ }^{81}$, B. Popov ${ }^{24}$, K. Porwit ${ }^{34}$, M. Posiadala-Zezula ${ }^{96}$, G. Pronost ${ }^{1,3}$, N.W. Prouse ${ }^{6}$, B. Quilain ${ }^{20}$, A. A. Quiroga ${ }^{57}$, E. Radicioni ${ }^{19}$, B. Radics ${ }^{85}$, P. J. Rajda ${ }^{97}$, J. Renner ${ }^{46}$, M. Rescigno ${ }^{10}$, G. Ricciardi ${ }^{36}$, B. Richards ${ }^{32}$, E. Rondio ${ }^{4}$, B. Roskovec ${ }^{84}$, S. Roth ${ }^{98}$, C. Rott ${ }^{79}$, A. Rubbia ${ }^{85}$, A.C. Ruggeri ${ }^{25}$, S. Russo ${ }^{24}$, A. Rychter ${ }^{35}$, D. Ryu ${ }^{83}$, K. Sakashita ${ }^{37,38}$, S. Samani ${ }^{16}$, F. Sánchez ${ }^{29}$, M. L. Sánchez ${ }^{27}$, S. Sano ${ }^{89}$, J. D. Santos ${ }^{27}$, G. Santucci ${ }^{22}$, P. Sarmah ${ }^{41}$, K. Sato ${ }^{88}$, M. Scott ${ }^{9}$, Y. Seiya ${ }^{99}$, T. Sekiguchi ${ }^{37,38}$, H. Sekiya ${ }^{1,2,3}$, J. W. Seo ${ }^{79}$, D. Sgalaberna ${ }^{85}$, Z. Shan ${ }^{30}$, A. Shaykina ${ }^{52}$, H. Shiba ${ }^{1}$, I. Shimizu ${ }^{68}$, K. Shimizu ${ }^{1}$, C. D. Shin ${ }^{77}$, M. Shinoki ${ }^{69}$, M. Shiozawa ${ }^{1,2,3}$, N. Skrobova ${ }^{7}$, K. Skwarczynski ${ }^{4}$, M.B. Smy ${ }^{23,2}$, J. Sobczyk ${ }^{91}$, H. W. Sobel ${ }^{23,2}$, F. J. P. Soler ${ }^{56}$, Y. Sonoda ${ }^{1}$, R. Spina ${ }^{19}$, B. Spisso ${ }^{100}$, P. Spradlin ${ }^{56}$, K. L. Stankevich ${ }^{81}$, L. Stawarz ${ }^{33}$, S. M. Stellacci ${ }^{100}$, A. I. Studenikin ${ }^{81}$, S. L. Suárez Gómez ${ }^{27}$, T. Suganuma ${ }^{69}$, S. Suvorov ${ }^{52}$, Y. Suwa ${ }^{101}$, A. T. Suzuki ${ }^{80}$, S. Y. Suzuki ${ }^{37,38}$, Y. Suzuki ${ }^{102}$, D. Svirida ${ }^{7}$, M. Taani ${ }^{30}$, M. Tada $^{37,38}$, A. Takeda ${ }^{1,2,3}$, Y. Takemoto ${ }^{1,2,3}$, A. Taketa ${ }^{11,3}$, Y. Takeuchi ${ }^{80,2}$, H. Tanaka ${ }^{1,2,3}$, H. I. Tanaka ${ }^{11,3}$, M. Tanaka ${ }^{37,38}$, T. Tashiro ${ }^{49,3}$, M. Thiesse ${ }^{70}$, L. F. Thompson ${ }^{70}$, D. Tiwari ${ }^{15}$, J. F. Toledo ${ }^{13}$, A. K. Tomatani-Sánchez ${ }^{103}$, G. Tortone ${ }^{25}$, K. M. Tsui ${ }^{39}$, T. Tsukamoto ${ }^{37,38}$, M. Tzanov ${ }^{82}$, Y. Uchida ${ }^{9}$, M. R. Vagins ${ }^{2,23,3}$, S. Valder ${ }^{32}$, V. Valentino ${ }^{19}$, G. Vasseur ${ }^{26}$, A. Vijayvargi ${ }^{90}$, W. G. S. Vinning ${ }^{32}$, D. Vivolo ${ }^{63}$, R. B. Vogelaar ${ }^{86}$, M. M. Vyalkov ${ }^{81}$, T. Wachala ${ }^{17}$, D. Wark ${ }^{16,51}$, M. O. Wascko 9 , R. A. Wendell ${ }^{8,2}$, J. R. Wilson ${ }^{30}$, S. Wronka ${ }^{4}$, J. Xia ${ }^{49}$, Z. Xie ${ }^{30}$, Y. Yamaguchi ${ }^{21}$, K. Yamamoto ${ }^{99}$, K. Yamauchi ${ }^{69}$, T. Yano ${ }^{1,3}$, N. Yershov ${ }^{52}$, M. Yokoyama ${ }^{5,2,3}$, M. Yonenaga ${ }^{69}$, J. Yoo ${ }^{104}$, I. Yu ${ }^{79}$, T. Zakrzewski ${ }^{4}$, B. Zaldivar ${ }^{58}$, J. Zalipska ${ }^{4}$, K. Zaremba ${ }^{35}$, G. Zarnecki ${ }^{4}$, M. Ziembicki ${ }^{35}$, K. Zietara ${ }^{93}$, M. Zito ${ }^{24}$, T Zhu ${ }^{9}$ and S. Zsoldos ${ }^{30}$

${ }^{1}$ University of Tokyo, Institute for Cosmic Ray Research, Kamioka Observatory, Kamioka, Japan. ${ }^{2}$ University of Tokyo, Kavli Institute for the Physics and Mathematics of the Universe (WPI), University of Tokyo Institutes for Advanced Study, Kashiwa, Japan. ${ }^{3}$ University of Tokyo, Next-generation Neutrino Science Organization, Kamioka, Japan. ${ }^{4}$ National Centre for Nuclear Research, Warsaw, Poland. ${ }^{5}$ University of Tokyo, Department of Physics, Tokyo, Japan. ${ }^{6}$ TRIUMF, Vancouver, British Columbia, Canada. ${ }^{7}$ P.N.Lebedev Physical Institute of the Russian Academy of Sciences, Moscow, Russia. ${ }^{8}$ Kyoto University, Department of Physics, Kyoto, Japan. ${ }^{9}$ Imperial College London, Department of Physics, London, United Kingdom. ${ }^{10}$ INFN Sezione di Roma, Università Sapienza, Dipartimento di Fisica, Roma, Italy. ${ }^{11}$ University of Tokyo, Earthquake Research Institute, Tokyo, Japan. ${ }^{12}$ Kyiv National University, Department of Nuclear Physics, Kyiv, Ukraine. ${ }^{13}$ Universitat Politècnica de València, Instituto de Instrumentaciòn para Imagen Molecular (i3M), Valencia, Spain. ${ }^{14}$ Laboratorio Subterráneo de Canfranc, Canfranc-Estación, Spain. ${ }^{15}$ University of Regina, Department of Physics, Regina, Saskatchewan, Canada. ${ }^{16}$ Oxford University, Department of Physics, Oxford, United Kingdom. ${ }^{17} \mathrm{H}$. Niewodniczański Institute 
of Nuclear Physics PAN, Cracow, Poland. ${ }^{18}$ INFN Sezione di Padova and Università di Padova, Dipartimento di Fisica, Padova, Italy. ${ }^{19}$ INFN Sezione di Bari and Università e Politecnico di Bari,Bari Italy. ${ }^{20}$ Ecole Polytechnique, IN2P3-CNRS, Laboratoire LeprinceRinguet, Palaiseau, France. ${ }^{21}$ Tokyo Institute of Technology, Department of Physics, Tokyo, Japan. ${ }^{22}$ York University, Department of Physics and Astronomy, Toronto, Ontario, Canada. ${ }^{23}$ University of California, Irvine, Department of Physics and Astronomy, Irvine, California, USA. ${ }^{24}$ Laboratoire de Physique Nucleaire et de Hautes Energie, IN2P3/CNRS, Sorbonne Universitè, Paris, France. ${ }^{25}$ INFN Sezione di Napoli, Napoli, Italy. ${ }^{26}$ IRFU, CEA, Universitè Paris-Saclay, Gif-sur-Yvette, France. ${ }^{27}$ University of Oviedo, Applied Mathematical Modeling Group/Department of Physics, Oviedo, Spain. ${ }^{28}$ University of Victoria, Department of Physics and Astronomy, Victoria, British Columbia, Canada. ${ }^{29}$ University of Geneva, Section de Physique, DPNC, Geneva, Switzerland. ${ }^{30}$ King's College London, Department of Physics, Strand Building, Strand, London, United Kingdom. ${ }^{31}$ S. N. Bose National Centre for Basic Sciences, Salt Lake City, Kolkata, India. ${ }^{32}$ University of Warwick, Department of Physics, Coventry, United Kingdom. ${ }^{33}$ Università degli Studi di Salerno and INFN Gruppo Collegato di Salerno, Fisciano, Italy. ${ }^{34}$ University of Silesia in Katowice, A. Chełkowski Institute of Physics, Poland. ${ }^{35}$ Warsaw University of Technology, Institute of Radioelectronics and Multimedia Technology, Warsaw, Poland. ${ }^{36}$ INFN Sezione di Napoli and Università Federico II di Napoli, Dipartimento di Fisica, Napoli, Italy. ${ }^{37}$ High Energy Accelerator Research Organization (KEK), Tsukuba, Japan. ${ }^{38}$ J-PARC Center, Tokai, Japan. ${ }^{39}$ University of Liverpool, Department of Physics, Liverpool, United Kingdom. ${ }^{40}$ University of Zaragoza, Centro de Astropartículas y Física de Altas Energías (CAPA), Zaragoza, Spain. ${ }^{41}$ Indian Institute of Technology Guwahati, Guwahati, India. ${ }^{42}$ Dongshin University, Laboratory for High Energy Physics, Naju, Korea. ${ }^{43}$ KTH Royal Institute of Technology, Department of Physics, Stockholm, Sweden. ${ }^{44}$ INFN Laboratori Nazionali di Legnaro, Legnaro (PD), Italy. ${ }^{45}$ Universidad Autonoma de Sinaloa, Culiacan, Mexico. ${ }^{46}$ Universitat de Santiago de Compostela, Instituto Gallego de Física de Altas Enerxías, Santiago de Compostela, Spain. ${ }^{47}$ Universidad de Guadalajara, CUCEI, Departamento de Fisica, Guadalajara, Jal., Mexico. ${ }^{48}$ Universidad de Guadalajara, CUCEA, IT.Ph.D. program, Guadalajara, Jal., Mexico. ${ }^{49}$ University of Tokyo, Institute for Cosmic Ray Research, Research Center for Cosmic Neutrinos, Kashiwa, Japan. ${ }^{50}$ Lancaster University, Physics Department, Lancaster, United Kingdom. ${ }^{51}$ STFC, Rutherford Appleton Laboratory, Harwell Oxford, and Daresbury Laboratory, Warrington, United Kingdom. ${ }^{52}$ Institute for Nuclear Research of the Russian Academy of Sciences, Moscow, Russia. ${ }^{53}$ Vishwakarma Institute of Information Technology, Pune, India. ${ }^{54}$ Tezpur University, Department of Physics, Sonitpur, India. ${ }^{55}$ University of Edinburgh, School of Physics and Astronomy, Edinburgh, United Kingdom. ${ }^{56}$ University of Glasgow, School of Physics and Astronomy, Glasgow, United Kingdom. ${ }^{57}$ Pontifícia Universidade Católica do Rio de Janeiro, Departamento de Física, Rio de Janeiro, Brazil. ${ }^{58}$ University Autonoma Madrid, Department of Theoretical Physics, Madrid, Spain. ${ }^{59}$ Donostia International Physics Center and Ikerbasque Foundation, Basque Country, Spain. ${ }^{60}$ Stockholm University, Oskar Klein Centre and Department of Physics, Stockholm, Sweden. ${ }^{61}$ Keio University, Department of Physics, Yokohama, Japan. ${ }^{62}$ Miyagi University of Education, Department of Physics, Sendai, Japan. ${ }^{63}$ Università della Campania "L. Vanvitelli" and INFN Sezione di Napoli, Napoli, Italy. ${ }^{64}$ Carleton University, Department of Physics, Ottawa, Ontario, Canada. ${ }^{65}$ Okayama University, Department of Physics, Okayama, Japan. ${ }^{66}$ Tohoku University, Department of Physics, Sendai, Japan. ${ }^{67}$ Institute for Theoretical Physics and Modeling, Yerevan, Armenia. ${ }^{68}$ Tohoku University, Research Center for Neutrino Science, Sendai, Japan. ${ }^{69}$ Tokyo University of Science, Department of Physics, Chiba, Japan. ${ }^{70}$ University of Sheffield, Department of Physics and Astronomy, Sheffield, United Kingdom. ${ }^{71}$ Nagoya University, Kobayashi-Maskawa Institute for the Origin of Particles and the Universe, Nagoya, Japan. ${ }^{72}$ Nagoya University, Institute for Space-Earth Environmental Research, Nagoya, Japan. ${ }^{73}$ SOKENDAI (The Graduate University for Advanced Studies), Tokai, Japan. ${ }^{74}$ University of Winnipeg, Department of Physics, Winnipeg, Manitoba, Canada. ${ }^{75}$ GIST College, Gwangju Institute of Science and Technology, Gwangju, Korea. ${ }^{76}$ Kyungpook National University, Department of Physics, Daegu, Korea. ${ }^{77}$ Chonnam National University, Department of Physics, Gwangju, Korea. ${ }^{78}$ Tokyo Metropolitan University, Department of Physics, Tokyo, Japan. ${ }^{79}$ Sungkyunkwan University, Department of Physics, Suwon, Korea. ${ }^{80}$ Kobe University, Department of Physics, Kobe, Japan. ${ }^{81}$ Moscow State University, Department of Theoretical Physics, Moscow, Russia. ${ }^{82}$ Louisiana State University, Department of Physics and Astronomy, Baton Rouge, Louisiana, USA ${ }^{83}$ Ulsan National Institute of Science and Technology, Department of Physics, Ulsan, Korea. ${ }^{84}$ Charles University, IPNP, FMF, Prague, Czech. ${ }^{85}$ ETH Zurich, Institute for Particle and Astroparticle Physics, Zurich, Switzerland. ${ }^{86}$ Virginia Tech, Center for Neutrino Physics, Blacksburg, Virginia, USA. ${ }^{87}$ University of Toronto, Department of Physics, Toronto, Ontario, Canada. ${ }^{88}$ Nagoya University, Graduate School of Science, Nagoya, Japan. ${ }^{89}$ Yokohama National University, Faculty of Engineering, Yokohama, Japan. ${ }^{90}$ Indian Institute of Technology Jodhpur, Department of Physics, Karwar, Rajasthan, India. ${ }^{91}$ Wroclaw University, Faculty of Physics and Astronomy, Wroclaw, Poland. ${ }^{92}$ Stockholm University, Oskar Klein Centre and Department of Astronomy, Stockholm, Sweden. ${ }^{93}$ Astronomical Observatory of the Jagiellonian University, Krakow, Poland. ${ }^{94}$ Uppsala University, Department of Physics and Astronomy, Uppsala, Sweden. ${ }^{95}$ British Columbia Institute of Technology, Physics Department, Burnaby, British Columbia, Canada. ${ }^{96}$ University of Warsaw, Faculty of Physics, Warsaw, Poland. ${ }^{97}$ AGH University of Science and Technology, Faculty of Computer Science, Electronics and Telecommunications, Krakow, Poland. ${ }^{98}$ RWTH Aachen University, III. Physikalisches Institut, Aachen, Germany. ${ }^{99}$ Osaka City University, Department of Physics, Osaka, Japan. ${ }^{100}$ INFN Gruppo Collegato di Salerno, Fisciano, Italy. ${ }^{101}$ Kyoto Sangyo University, Department of Astrophysics and Atmospheric Sciences, Kyoto, Japan. ${ }^{102}$ University of Tokyo, Tokyo, Japan. ${ }^{103}$ Tecnologico de Monterrey, Escuela de Ingenieria y Ciencias, Zapopan, Jalisco, Mexico. ${ }^{104}$ Korea Institute of Science and Technology, Department of Physics, Daejeon, Korea.

${ }^{*}$ The current affiliation is Indian Institute of Technology Kharagpur, Department of Physics, Kharagpur, India. 